\title{
FRECUENCIA DEL TUMOR VENÉREO TRANSMISIBLE EN CANINOS: CASUÍSTICA DEL LABORATORIO DE PATOLOGÍA VETERINARIADE LA UNIVERSIDAD NACIONAL MAYOR DE SAN MARCOS (PERIODO 1998 - 2004)
}

\author{
Frequency of the Transmissible Venereal Tumour in Dogs: Caseload in the \\ Pathology Laboratory of the National University of San Marcos (Period \\ 1998-2004)
}

\author{
Narda Mendoza V. ${ }^{1}$, Alfonso Chavera C. ${ }^{1,2}$, Néstor Falcón P. ${ }^{3,4}$, Rosa Perales C. ${ }^{1}$
}

\section{RESUMEN}

El presente estudio se realizó para determinar la frecuencia del Tumor Venéreo Transmisible (TVT) en canes de la ciudad de Lima durante el periodo 1998-2004 y establecer su relación con el sexo, edad y raza; así como determinar las localizaciones más frecuentes de las neoplasias. Se analizaron los registros de citología e histopatología del Laboratorio de Patología de la Facultad de Medicina Veterinaria de la Universidad Nacional Mayor de San Marcos. Se encontró una frecuencia de TVT de 8.4\% (78/925), no existiendo predisposición de sexo. Los animales más afectados fueron de raza cruzada y los que tenían entre 1 y 5 años de edad. La localización más frecuente del tumor fue el genital externo (prepucio o pene en los machos y la vulva o vagina en las hembras); sin embargo, también se pudo observar localizaciones extragenitales como en piel, mucosas nasal, oral y anal, ganglio inguinal y bazo.

Palabras clave: tumor venéreo transmisible, frecuencia, canino, neoplasia

\section{Abstract}

The objective of this study was to determine the frequency of Transmissible Venereal Tumour (TVT) in dogs from Lima city, during 1998 to 2004, to establish the relationship with sex, breed, and age, and to determine the organs most affected by the tumours. The cytology and histopathology records of the Pathology Laboratory (School of Veterinary Medicine, Universidad Nacional Mayor de San Marcos) were analyzed. The frequency of TVT was $8.4 \%$ (78/925), and without sex effect. Crossbred dogs and dogs of one to five years of age were the most affected. The external genitalia were the most affected site (prepuce or penis in males and vulva or vagina in females). Extra genital lesions in the nasal, oral, and anal mucosa, skin, inguinal lymph nodules and spleen were also observed.

Key words: transmissible venereal tumour, frequency, canine, neoplasia

\footnotetext{
${ }^{1}$ Laboratorio de Histología, Embriología y Patología Veterinaria, ${ }^{3}$ Laboratorio de Medicina Veterinaria Preventiva, Facultad de Medicina Veterinaria, Universidad Nacional Mayor de San Marcos, Lima

${ }^{2}$ E-mail: achaverac@unmsm.edu.pe

${ }^{4}$ Dirección actual: Facultad de Veterinaria y Zootecnia, Universidad Peruana Cayetano Heredia, Lima
} 


\section{INTRODUCCIÓN}

El tumor venéreo transmisible (TVT) es una neoplasia que se transmite a través del coito, mediante implantaciones de células tumorales, por lo que es común encontrarlo en los genitales externos; sobre todo en canes con alta actividad sexual (Jubb et al., 1992; Rogers, 1997; Scott et al., 2002; Ferreira, 2003, Nak et al., 2005). Sin embargo, también se presentan implantaciones extragenitales, producto de mordeduras, rascado, lamido u olfateo directo de la zona del tumor; ya sea de un animal enfermo a otro sano o por un autotransplante a partir del tumor primario (Moulton, 1978; Trigo, 1998; Ferreira, 2003).

El TVT tiene una alta prevalencia en ciudades de países en desarrollo con gran densidad de habitantes, en donde existe una alta concentración de perros vagabundos y un pobre control de la crianza (Scott et al., 2002). En países como Uruguay, Kenia y Tanzania se reportan frecuencias de 7.5, 12 y 34\%, respectivamente (Ndiritu et al., 1977; Batamuzi et al., 1992; Caponi et al., 1999). Dentro de las neoplasias de origen misceláneo y de localización genital, el TVT es la entidad que, desde mediados del siglo XX, ha sido recurrentemente reportada en canes de la ciudad de Lima (Takano, 1964; Rodríguez, 1978; Castro, 1985; Díaz VM, 1991; Díaz PL, 1993; Clavo, 1995; San Martín, 2005), y en otras ciudades de América Latina (Trigo, 1993; Ferreira, 2003).

No se ha hallado una clara predisposición sexual (Rogers, 1997; Scott et al., 2002), aunque hay reportes que demuestran diferencia a favor de las hembras (Clavo, 1995; Sousa et al., 2000). Tampoco se ha encontrado una predisposición racial (Rogers, 1997; Scott et al., 2002).

En 1995 se realizó el último estudio poblacional sobre el TVT en la ciudad de Lima, de modo que el presente trabajo tuvo como objetivo establecer la frecuencia del
TVT canino, sus tendencias en el tiempo, y su relación con la edad, sexo, raza, ubicación del tumor y lugar de procedencia del animal.

\section{MATERIALES Y MÉTODOS}

El estudio se llevó acabo en el Laboratorio de Histología, Embriología y Patología Veterinaria de la Facultad de Medicina Veterinaria de la Universidad Nacional Mayor de San Marcos. Se revisaron los archivos de patología (protocolos de necropsia, histopatología y citología), comprendidos entre los años 1998 y 2004 para obtener la casuística de TVT diagnosticados por citología o histopatología.

Las variables en estudio fueron la raza (pura y cruzada), edad ( $<1,1-5,>5-9,>9$ años), sexo, localización del tumor (genital, primaria extragenital, mixta) y lugar de procedencia del animal (Lima Norte, Lima Sur, Lima Este, Lima Moderna, Lima Antigua, Provincia Constitucional del Callao).

La frecuencia del TVT, durante el periodo 1998-2004, se estableció en base a los casos positivos a TVT y al total de neoplasias caninas. La asociación entre las variables de clasificación y la presencia de TVT se evaluó mediante la prueba de Chi Cuadrado.

\section{Resultados}

En el periodo 1998-2004 se registraron 2742 protocolos de necropsia, histopatología y citología en caninos registrados en el laboratorio de patología; de estos, 925 casos (33.7\%) fueron procesos neoplásicos, y dentro de ellos, 78 casos correspondieron a TVT (8.4\% del total de neoplasias).

Las razas en las que se encontró la neoplasia fueron Bóxer, Cocker, Collie, Doberman, Dogo Argentino, Labrador, Pastor Alemán, Pequinés, Salchicha, y Samoyedo, así como en canes cruzados. 
Cuadro 1. Frecuencia del Tumor Venéreo Transmisible canino, de acuerdo a la casuística del Laboratorio de Histología, Embriología y Patología Veterinaria de la Facultad de Medicina Veterinaria de la Universidad Nacional Mayor de San Marcos (Periodo 1998-2004), según sexo, edad y grupo racial

\begin{tabular}{llccc}
\hline \multirow{3}{*}{ Sexo } & Variables & $\begin{array}{c}\text { Total de } \\
\text { neoplasias } \\
(\mathrm{n})\end{array}$ & $\begin{array}{c}\text { Tumor Venéreo } \\
\text { Transmisible } \\
(\mathrm{n})\end{array}$ & $\begin{array}{c}\text { Frecuencia } \\
(\%)\end{array}$ \\
& Hembra & 459 & 42 & 9.1 \\
& Macho & 416 & 33 & 7.9 \\
& No registra & 50 & 3 & 6.0 \\
& $<1$ & 53 & 5 & 9.4 \\
Edad & $1-5$ & 222 & 42 & 18.9 \\
& $>5-9$ & 298 & 19 & 6.4 \\
& $>9$ & 289 & 5 & 1.7 \\
& No registra & 63 & 7 & 11.1 \\
\multirow{2}{*}{ Raza } & Pura & 604 & 24 & 4.0 \\
& Cruzada & 269 & 52 & 19.3 \\
\hline Total & No registra & 52 & 2 & 3.9 \\
\hline
\end{tabular}

La distribución de los casos de TVT se encuentra en el Cuadro 1. La edad de los animales afectados se encontró entre los siete meses hasta los 13 años de edad. Los casos con localización primaria extragenital fueron siete en mucosa nasal, dos en mucosa oral, una en mucosa anal y una en el párpado. Aquellos con localización tanto genital como extragenital presentaron lesiones en pene o vulva, labio, piel, ganglio inguinal y bazo.

El 80.5\% de los casos de TVT tuvieron una localización genital, el $14.3 \%$ fue extragenital y el 5.2\% se presentó en ambas zonas.

\section{Discusión}

La frecuencia hallada de TVT fue de $8.4 \%$. No obstante, se debe tener en consideración que parte de la población canina no está sujeta a la observación clínica, ni tiene acceso al diagnóstico citológico o histopatológico de las neoformaciones que podrían presentar, siendo muchas veces tratado sin previo diagnóstico microscópico; por lo tanto, es de esperar que la frecuencia observada en este análisis pueda estar afectado por otros factores. Sin embargo, este porcentaje es relativamente alto y estaría influenciado por descuido o ignorancia en la crianza de mascotas, así como por una elevada presencia de canes callejeros, ya sea en estado de abandono o por el poco control por parte sus dueños, quienes permiten que el animal permanezca gran parte del día en la vía pública, teniendo un estrecho contacto con otros animales y ocurran cópulas indiscriminadas.

La frecuencia de TVT obtenida en el presente trabajo es muy superior a lo reportado en países europeos (Smith y Jones, 1962, $0.93 \%$ ); sin embargo, es similar a otros reportes latinoamericanos (Ndiritu et al., 1977; 
Caponi et al., 1999) e inferior al 34\% reportado en Tanzania (Batamuzi et al., 1992). No obstante, la frecuencia hallada en el presente estudio no parece haber variado significativamente en Lima en relación a trabajos previos, aunque es ligeramente inferior a las frecuencias reportadas por Takano (1964), Rodríguez (1978), Castro (1985), Díaz VM (1991) y San Martín (2005).

Esta neoplasia se presentó indistintamente en canes machos y hembras (Cuadro 1). Los canes, indistintamente de su sexo, están propensos a presentar injurias genitales durante la actividad sexual y son, por lo tanto, susceptibles al transplante de células tumorales (Moulton, 1978). Similares conclusiones fueron encontradas por Gurel et al. (2002) y Ortega et al. (2003) aunque hay otros reportes que señalan una mayor frecuencia en hembras (Clavo, 1995; Sousa et al., 2000).

Los perros más afectados tenían edades entre 1 a 5 años, probablemente por la mayor actividad sexual que desempeñan a esas edades (Díaz PL, 1993; Clavo, 1995; Caponi et al., 1999; Sousa et al., 2000; Gurel et al., 2002; Ortega et al., 2003). Esto significa que la edad juega un papel importante, ya que desde que el animal llega a la pubertad y tiene contacto sexual, está propenso al contagio de la enfermedad.

Los canes cruzados fueron los más afectados (19.3\%), probablemente debido a que son los que mayoritariamente se pueden observar deambulando en las calles. Los canes de raza son, por lo general, mejor cuidados $y$, según el historial de algunos de ellos, se contagiaron de la neoplasia al extraviarse de casa o por una monta autorizada con un perro de su misma raza, sin que estos canes hallan sido previamente inspeccionados por un Médico Veterinario.

Debido a que la principal forma de transmisión del TVT es mediante el apareamiento, la localización genital (vulva o vagina en las hembras y pene en los machos) fue la de mayor frecuencia (79.5\%), coincidiendo con otros reportes (Ndiritu et al., 1977; Gurel et al., 2002).

Los 11 casos de TVT extragenital como lesión primaria (14.3\%) constituyen el primer reporte en el país de la ocurrencia extragenital de esta neoplasia. La mucosa nasal fue el principal asiento del TVT (siete canes), aunque también se encontró en párpado y en la mucosa oral y anal. Estas implantaciones extragenitales son poco frecuentes y se dan como resultado del comportamiento social que tienen los perros de lamer u olfatear los genitales (Papazoglou et al., 2001; Gurel et al., 2002). La destrucción o erosión de las mucosas o piel tiene un importante impacto en la formación del tumor en regiones extragenitales.

El caso de TVT en la mucosa anal podría deberse al lamido de perros infectados o a los intentos de monta por parte de un macho con TVT genital, situaciones que se dan, incluso entre un macho dominante con otros machos (Ortega et al., 2003). Así mismo, Boscos et al. (1998) reportan un caso de TVT ocular primario, ocurrido posterior a la pelea con un perro callejero, donde tuvo contacto con las secreciones contaminadas del can enfermo.

En los canes con afección genital y extragenital del tumor, se presume que la localización primaria fue la genital, y de allí se dieron implantaciones extragenitales o metástasis en zonas como piel, labios, ganglio linfático inguinal y bazo. Estas metástasis se encontraron en un 5.2\% (4 casos), porcentaje similar a lo reportado en la literatura (Moulton, 1978; Trigo, 1993; Rogers, 1997; Scott et al., 2002). La transmisión del tumor a los labios y varias regiones de la piel se pudo haber facilitado por acción del rascado y lamido (Boscos et al., 1998; Gurel et al., 2002). La metástasis al ganglio inguinal se debe a la vía linfática (Pereira et al., 2000).

La mayoría de los casos provinieron de las afueras de la ciudad donde se observa 
una alta concentración de perros callejeros, situación similar a la observada por Caponi et al. (1999). Estos canes sirven de reservorio del TVT y probablemente también de otras enfermedades, que pueden ser transmitidas a canes cuyos propietarios los pasean por los parques públicos o que tienen libre acceso a exteriores (Ortega et al., 2003).

Se hace necesario que los médicos veterinarios lleven un registro de casos, con datos completos del animal, una minuciosa observación clínica y resultados de laboratorio, de manera que se tenga una base de datos de las enfermedades presentes en el medio, que serviría para la realización de trabajos futuros.

\section{Conclusiones}

- En el periodo 1998-2004 se diagnosticaron 78 casos de Tumor Venéreo Transmisible (TVT) en caninos, de un total de 925 neoplasias caninas, lo que equivale a una frecuencia de $8.4 \%$.

- El TVT se presentó indistintamente en canes hembras y machos, y con mayor frecuencia en perros cruzados y con edades entre uno y cinco años.

- La localización genital se presentó en el $79.4 \%$ de los casos.

\section{Literatura Citada}

1. Batamuzi EK, Kasuku AA, Agger JF. 1992. Risk factors associated with canine transmissible venereal tumour in Tanzania. Prev Vet Med 13: 13-17.

2. Boscos CM, Ververidis HN, Tondis DK, Stamou AI, Samartzi FC. 1998. Ocular involvement of transmissible venereal tumour in a dog. Vet Ophthal 1: 167-170.

3. Caponi O, Vitale E, Sallúa S. 1999. Tumores en la especie canina. Bol SICLIVET, Montevideo 2(6): 1-3.
4. Castro SJ. 1985. Frecuencia en la presentación de neoplasias en caninos. Periodo 1978-1983. Tesis de Médico Veterinario. Lima: Facultad de Medicina Veterinaria, Univ. Nacional Mayor de San Marcos. 46 p.

5. Clavo F. 1995. Neoplasias de los órganos genitales de la perra. Estudio estadístico años 1973-1994. Tesis de Médico Veterinario. Lima: Facultad de Medicina Veterinaria, Univ. Nacional Mayor de San Marcos. 43 p.

6. Díaz VM. 1991. Procesos neoplásicos en caninos. Análisis estadístico. Periodo 1984-1989. Tesis de Médico Veterinario. Lima: Facultad de Medicina Veterinaria, Univ. Nacional Mayor de San Marcos. 28 p.

7. Díaz PL. 1993. Neoplasias del tracto genital del perro - Estudio estadístico. Tesis de Médico Veterinario. Lima: Facultad de Medicina Veterinaria, Univ. Nacional Mayor de San Marcos. 34 p.

8. Ferreira G. 2003. Patología veterinaria. Colombia: Universidad de Antioquia. $622 \mathrm{p}$.

9. Gurel A, Kuscu B, Gulanber E, Arun S. 2002. Transmissible venereal tumours detected in the extragenital organs of dogs. Israel J Vet Med 57(2): 30-34.

10. Jubb K, Kennedy P, Palmer N. 1992. Pathology of domestic animals. $4^{\text {th }}$ ed. USA: Academic Press. Vol. 3. 653 p.

11. Moulton JE. 1978. Tumours in domestic animals. $2^{\text {nd }}$ ed. Los Angeles, USA: University of California. $465 \mathrm{p}$.

12. Nak D, Nak Y, Cangul I, Tuna B. 2005. A clinic-pathological study on the effect of vincristine on transmissible venereal tumours in dogs. J Vet Med A 19: 366-370.

13. Ndiritu CG Mbogwa SW, Sayer PD. 1977. Extragenital localization of transmissible venereal tumour in dogs. Mod Vet Pract 58: 940-946.

14. Ortega PA, Acebedo AM, Sauri AC, Bolio GM, Gutiérrez BE. 2003. Prevalencia de tumor venéreo transmisible en perros callejeros en la ciudad de Mérida, Yucatán, México. Rev Biomédica 14 (2): 83-87. 
15. Papazoglou LG Koutinas AF, Plevraki AG Tontis D. 2001. Primary intranasal transmissible venereal tumour in the dog: A retrospective study of six spontaneous cases. J Vet Med A 48: 391-400.

16. Pereira J, Silva A, Martins A, Ferreira A, Brooks DE. 2000 Immunohistochemical characterization of intraocular metastasis of a canine transmissible venereal tumour. Vet Ophthal 3: 43-47.

17. Rodríguez GJ. 1978. Neoplasias en la especie canina: Estudio estadístico de 301 casos entre 1964-1977. Tesis de Médico Veterinario. Lima: Facultad de Medicina Veterinaria, Univ. Nacional Mayor de San Marcos. 35 p.

18. Rogers KS. 1997. Transmissible venereal tumour. Comp Cont Educ Pract Vet 19: 1036-1045.

19. San Martín M. 2005. Neoplasias caninas: Evaluación estadística. Periodo 1990-1995. Tesis de Médico Veterinario. Lima: Facultad de Medicina Veterinaria, Univ. Nacional Mayor de San Marcos. 28 p.
20. Scott WD, Miller HW, Griffin EC. 2002. Muller \& Kirk. Dermatología en pequeños animales. $6^{\text {ta }}$ ed. Buenos Aires: Ed. Inter-Médica. 1572 p.

21. Smith H, Jones T. 1962. Patología veterinaria. $2^{\text {da }}$ ed. México: Ed. Uteha. $1061 \mathrm{p}$.

22. Sousa J, Saito V, Nardi A, Rodaski S, Guérios S, Bacila M. 2000. Características e incidência do tumor venéreo transmissível (TVT) en câes e eficiência da quimioterapia e outros tratamentos. Arch Vet Sci, Brasil 5: 41-48.

23. Takano MF. 1964. Estudio retrospectivo de 154 procesos neoplásicos en la especie canina. Tesis de Médico Veterinario. Lima: Facultad de Medicina Veterinaria, Univ. Nacional Mayor de San Marcos. 55 p.

24. Trigo F. 1993. Patología general veterinaria. $2^{\text {da }}$ ed. México: Ed. McGraw-Hill Interamericana. $242 \mathrm{p}$.

25. Trigo F. 1998. Patología sistémica veterinaria. $3^{\text {ra }}$ ed. México: Ed. McGrawHill Interamericana. 421 p. 